\title{
Heating of coronal active regions
}

\author{
Daniel O. Gómez ${ }^{1,2}$ \\ ${ }^{1}$ Instituto de Astronomía y Física del Espacio, C.C. 67 - Suc. 28, \\ (1428) Buenos Aires, Argentina - email: gomez@iafe.uba.ar \\ ${ }^{2}$ Departamento de Física, Facultad de Ciencias Exactas y Naturales (UBA), \\ Ciudad Universitaria, (1428) Buenos Aires, Argentina
}

\begin{abstract}
Recent observations of coronal loops in solar active regions show that their heating must be a truly dynamic process. Even though it seems clear that the energy source is the magnetic field that confines the coronal plasma, the details of how it dissipates are still a matter of debate. In this presentation we review the theoretical models of coronal heating, which have been traditionally clasified as DC or AC depending on the electrodynamic response of the loops to the photospheric driving motions.

Also, we show results from numerical simulations of the internal dynamics of coronal loops within the framework of the reduced MHD approximation. These simulations indicate that the application of a stationary velocity field at the photospheric boundary leads to a turbulent stationary regime after several photospheric turnover times. Once this turbulent regime is set, both $\mathrm{DC}$ and $\mathrm{AC}$ stresses dissipate at faster rates as a result of a direct energy cascade.
\end{abstract}

Keywords. Sun: corona, Sun: X-rays, magnetic fiels, MHD, turbulence.

\section{Introduction}

Theoretical models of coronal heating have been traditionally classified into two broad categories, according to the time scales involved in the driving at the loop bases: (a) AC or wave models, for which the energy is provided by waves at the Sun's photosphere, with timescales much faster than the time it takes an Alfven wave to cross the loop; (b) DC or stress models, which assume that energy dissipation takes place by magnetic stresses driven by slow footpoint motions (compared to the Alfven wave crossing time) at the Sun's photosphere. Although these scenarios seem mutually exclusive, two common factors prevail: (i) the ultimate energy source is the kinetic energy of the subphotospheric velocity field, (ii) the existence of fine scale structure is essential to speed up the dissipation mechanisms invoked.

Review articles on coronal heating (Narain \& Ulmschneider 1990; Gómez 1990; Zirker 1993; Narain \& Ulmschneider 1996) explore the theoretical models in further detail. More recent reviews can be found in Mandrini et al. (2000); Demoulin et al. (2003); Aschwanden (2004), where observational tests of the models are also described.

A natural candidate for the dissipation of the energy provided by subphotospheric motions is Joule heating, but the typical time scale to dissipate coronal magnetic stresses at the length scale of the driving motions is exceedingly long. This time scale can be estimated as $l^{2} / \eta \approx 10^{6}$ years ( $l$ is a typical length scale an $\eta$ is the plasma resistivity). Most of the theories of coronal heating invoke different mechanisms to speed up energy dissipation (Parker 1972, 1988; Heyvaerts \& Priest 1992; van Ballegooijen 1986; Mikić et al. 1989; Longcope \& Sudan 1994; Hendrix \& van Hoven 1996; Galsgaard \& Nordlund 1996; Gudiksen \& Nordlund 2002).

One of the proposed scenarios to speed up dissipation is the assumption that the magnetic and velocity fields of the coronal plasma are in a turbulent state (Gómez \& Ferro 
Fontán 1988, 1992; Heyvaerts \& Priest 1992). On these models, turbulent fluctuations of both fields are predominantly in the directions perpendicular to the main magnetic field. In a turbulent regime, energy is transferred from photospheric motions to the magnetic field and then cascades toward small scales due to nonlinear interactions, until highly structured electric currents are formed. The development of fine scales (i.e. the drastic reduction of $l$ ) to enhance the dissipation of either waves or DC currents is a natural outcome of turbulence models.

\section{Reduced MHD}

To theoretically describe the dynamics of coronal loops in solar (or stellar) active regions, we assume these loops to be relatively homogeneous bundles of fieldlines, with their footpoints deeply rooted into the photosphere. Subphotospheric convective motions move individual fieldlines around, generating magnetic stresses in the coronal portion of the loop. More specifically, we consider a simplified model of a coronal magnetic loop with length $L$ and cross section $2 \pi l_{p} \times 2 \pi l_{p}$, where $l_{p}$ is the lengthscale of typical subphotospheric motions. For elongated loops, i.e. such that $2 \pi l_{p}<<L$, it seems reasonable to neglect toroidal effects. The main magnetic field $\mathbf{B}_{\mathbf{0}}$ is assumed to be uniform and parallel to the axis of the loop (the $z$ axis). The planes at $z=0$ and $z=L$ correspond to the photospheric footpoints.

Under these symplifying assumptions, we are able to use the reduced MHD approximation (Strauss 1976)), according to which the plasma moves incompressibly in planes perpendicular to the axial field $\mathbf{B}_{\mathbf{0}}$, and the transverse component of the magnetic field is small compared to $\mathbf{B}_{\mathbf{0}}$. The very high electric conductivity (frozen field) allows photospheric motions to easily drive magnetic stresses in the corona (Parker 1972), since the field lines twist and bend due to these motions, generating tranverse components of velocity $\mathbf{u}$ and magnetic field $\mathbf{b}$. Therefore:

$$
\begin{gathered}
\mathbf{B}=B_{0} \mathbf{z}+\mathbf{b}(x, y, z, t), \quad \mathbf{b} \cdot \mathbf{z}=0 \\
\mathbf{u}=\mathbf{u}(x, y, z, t), \quad \mathbf{u} \cdot \mathbf{z}=0
\end{gathered}
$$

Since both $\mathbf{b}$ and $\mathbf{u}$ are two-dimensional and divergence-free fields, they can be represented by scalar potentials:

$$
\begin{aligned}
& \mathbf{b}=\nabla \times(a \mathbf{z})=\nabla a(x, y, z, t) \times \mathbf{z} \\
& \mathbf{u}=\nabla \times(\psi \mathbf{z})=\nabla \psi(x, y, z, t) \times \mathbf{z}
\end{aligned}
$$

where $\nabla$ indicates derivatives in the $x, y$ plane. The reduced MHD equations for the stream function $\psi \mathbf{z}$ and the vector potential $a \mathbf{z}$ are:

$$
\begin{gathered}
\partial_{t} a=v_{A} \partial_{z} \psi+[\psi, a]+\eta \nabla^{2} a \\
\partial_{t} w=v_{A} \partial_{z} j+[\psi, w]-[a, j]+\nu \nabla^{2} w
\end{gathered}
$$

where $w=-\nabla^{2} \psi$ is the fluid vorticity, $j=-\nabla^{2} a$ is the current density, $v_{A}=B_{0} / \sqrt{4 \pi \rho}$ is the Alfvén velocity and $\rho$ is the plasma density.

Eqn 2.5 describes the advection of the potential $a$ and Eqn 2.6 corresponds to the evolution of vorticity $w$. The terms $v_{A} \partial_{z}$ represent the coupling between neighboring $z=$ constant planes and describe parallel wave propagation. The $\nabla^{2}$ terms represent dissipative effects, the constants $\eta$ and $\nu$ being the resistivity and viscosity coefficients. The nonlinear terms are those represented by the Poisson brackets $\left([A, B]=\partial_{x} A \partial_{y} B-\right.$ $\left.\partial_{y} A \partial_{x} B\right)$. Their role is to couple normal modes in such a way that energy, and other ideal invariants, can be transferred between them. 


\section{RMHD turbulence}

We numerically explore the feasibility of a turbulent scenario, describing the internal dynamics of coronal loops through the RMHD approximation. We assume periodicity for the lateral boundary conditions, and specify the velocity fields at the $z=0$ and $z=L$ photospheric boundaries. In particular, we assume $\psi(z=0)=0$ and $\psi(z=L)=\Psi(x, y)$ where the stream function $\Psi(x, y)$ describes stationary and incompressible footpoint motions on the photospheric plane. We specify the Fourier components of $\Psi(x, y)$ as $\Psi_{\mathbf{k}}=$ $\Psi_{0}$ inside the ring $3<l_{p}|k|<4$ on the Fourier plane, and $\Psi_{\mathbf{k}}=0$ elsewhere, to simulate a stationary and isotropic pattern of photospheric granular motions of diameters between $2 \pi l_{p} / 4$ and $2 \pi l_{p} / 3$. The strength $\Psi_{0}$ is proportional to a typical photospheric velocity $V_{p} \approx 1 \mathrm{~km} . \mathrm{s}^{-1}$. The typical timescale associated to these driving motions, is the eddy turnover time, which is defined as $t_{p}=l_{p} / V_{p} \approx 10^{3} \mathrm{sec}$. We choose a narrowband and non-random forcing to make sure that the broadband energy spectra and the signatures of intermittency that we obtained (see below) are exclusively determined by the nonlinear nature of the MHD equations.

To transform (2.5)-(2.6) into their dimensionless form, we choose $l_{p}$ and $L$ as the units for transverse and longitudinal distances $\left(l_{p} \approx 10^{3} \mathrm{~km}\right.$ and $\left.L \approx 10^{4}-10^{5} \mathrm{~km}\right)$ and $t_{A} \equiv L / v_{A}$ as the time unit $\left(t_{A} \approx 10-100 \mathrm{sec}\right)$.

In Figure 1 we show the results obtained from a simulation extending from $t=0$ to $t=100 t_{A}$. The upper panel shows the magnetic $\left(E_{B}\right.$, thin trace) and kinetic $\left(E_{U}\right.$, dotted line) energies, as well as the total energy $\left(E=E_{U}+E_{B}\right.$, thick trace). We can see that after about ten Alfven times, the energy reaches a stationary regime, since the work done by footpoint motions statistically (i.e. in time average) reaches an equilibrium with the dissipative processes (electric resistivity and fluid viscosity). In this stationary regime most of the energy is magnetic, while kinetic energy is only about $5 \%$ of the total. In the lower panel, we show the dissipation rate $(D$, thick trace) and the incoming Poynting flux $(P$, thin trace), showing that their time averages are approximately equal.

Gómez \& Ferro Fontán (1988) (also Gómez \& Ferro Fontán (1992)) showed that this stationary equilibrium corresponds to a turbulent regime (see also ?). The associated energy cascade bridges the gap between the large spatial scales where energy is injected, to the much smaller scales where it dissipates (see Dmitruk \& Gómez (1997)). The dependence of this mean dissipation rate $\epsilon=\langle D\rangle=\langle P\rangle(<\ldots>$ : time average)

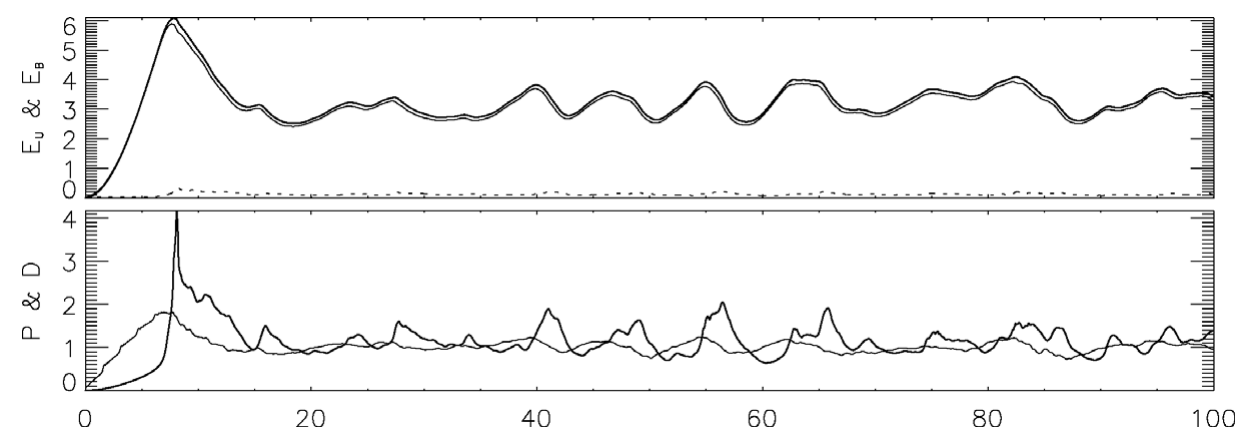

Figure 1. Time series obtained from simulations externally driven by a stationary velocity field. Upper panel: Magnetic energy (thin), kinetic energy (dotted) and total energy (thick). Lower panel: Energy dissipation rate (thick) and Poynting flux (thin). 
with the physical parameters of the loop is (Dmitruk \& Gómez 1999)

$$
\epsilon \propto \frac{\rho l_{p}^{2}}{t_{A}^{3}}\left(\frac{t_{A}}{t_{p}}\right)^{\frac{3}{2}}
$$

The kinetic and magnetic Reynolds numbers in our simulations $\left(R e=l_{p}^{2} /\left(\eta t_{A}\right)=R m\right)$ were carefully chosen to guarantee a proper resolution of the smallest spatial structures (i.e. the largest wavenumbers), where dissipation becomes dominant.

Another feature which can readily be observed in Figure 1 is the spiky nature of these time series, which is caused by the ubiquitous presence of intermittency in turbulent regimes. Dmitruk et al. (1998) (see also Georgoulis et al. (1998)) associated each of these spikes of energy dissipation with Parker's nanoflares (see Parker (1988)) and studied the statistical properties of these dissipation events. The main result from this statistical study (see also Gómez \& Dmitruk (2008)) is that the number of dissipation events (or nanoflares) as a function of their individual energies $N(E)$ follows a power law $N(E) \approx$ $E^{-3 / 2}$, which is remarkably comparable to the result obtained for larger dissipation events (such as microflares and flares), gathering a large number of observational studies and reported by Aschwanden (2004).

\section{Wave propagation and dissipation}

To study the response of coronal loops to waves being pumped at their footpoints, we performed RMHD simulations applying a time-periodic velocity field at $z=L$, with a time frequency $w_{0}$. In Figure 2 we show the results obtained for a relatively slow frequency $\omega_{0}=0.1$ (i.e. a wave period equal to ten loop Alfven times). In Figure 3 we show the results for the case $\omega_{0}=1.0$. Note that both the energy and dissipation rate reach maximum levels much larger than the previous case, which is to be expected for a resonant mode.

We repeat this type of RMHD simulations for a range of wave frequencies and compute the asymptotic value for the dissipation rate, which is shown in Figure 4 . This curve displays the expected behavior of an externally driven resonant system. The particular case of externally driven coronal loops was addressed analytically by Inverarity \& Priest (1995). The full line in Figure 4 shows the analytical result for the viscosity used in our simulations, i.e. $\nu=\eta=4.10^{-3}$. The dotted trace corresponds to the ideal case.

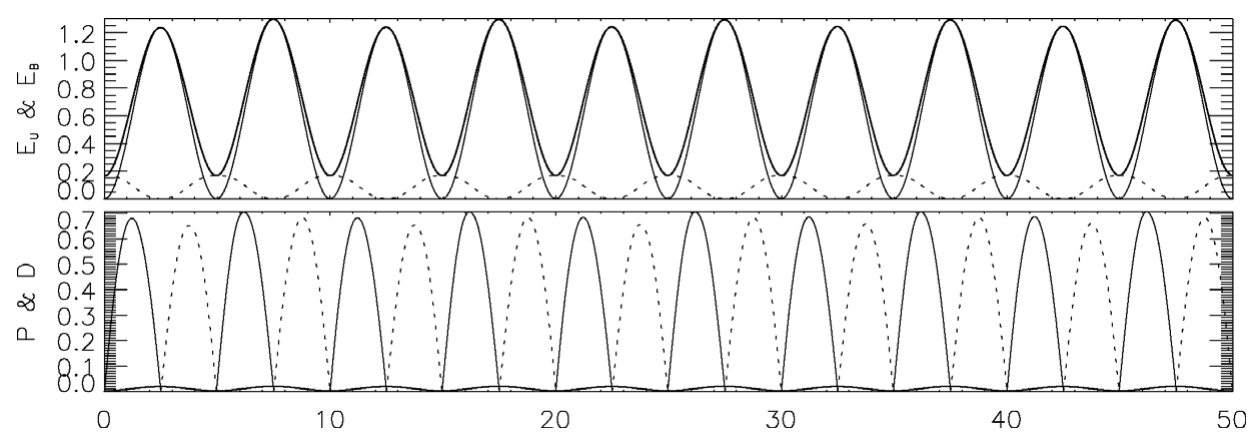

Figure 2. Time series obtained from simulations externally driven by a pulsating velocity field at the frequency $\omega_{0}=0.1$. Upper panel: Magnetic energy (thin), kinetic energy (dotted) and total energy (thick). Lower panel: Energy dissipation rate (thick), positive (thin) and negative (dotted) Poynting flux. 


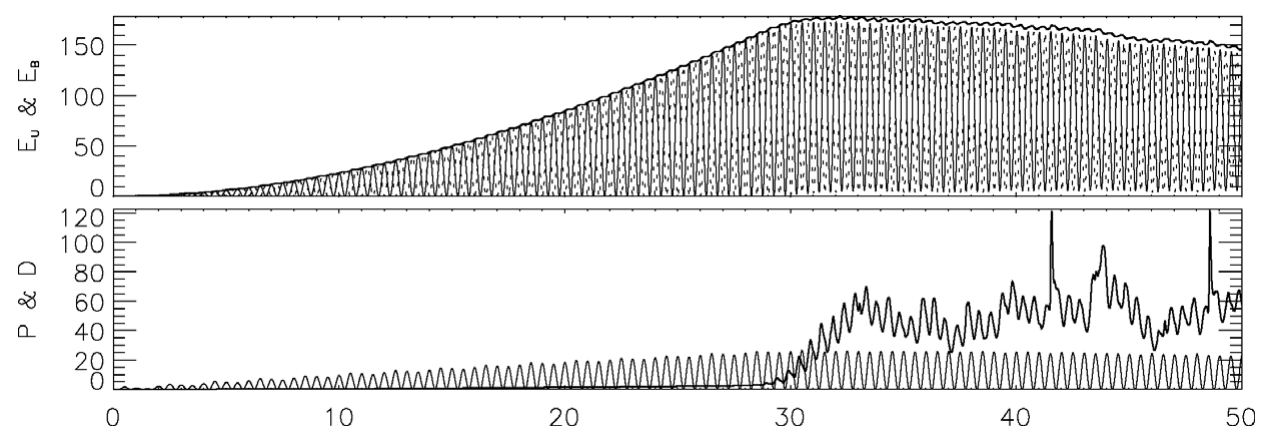

Figure 3. Time series obtained from simulations externally driven by a pulsating velocity field at the frequency $\omega_{0}=1.0$. Upper panel: Magnetic energy (thin), kinetic energy (dotted) and total energy (thick). Lower panel: Energy dissipation rate (thick), positive (thin) and negative (dotted) Poynting flux.

If the wave propagates in a turbulent flow, we would expect its dissipation to be enhanced with respect to the case of propagation in a laminar medium. We therefore performed the following combined simulations: drive the loop with the stationary motions described in $\S 3$ until reaching a stationary turbulent regime, and then add the wave pumping boundary motion at a given frequency $\omega_{0}$ described in this section. In Figure 5 , we show the asymptotic dissipation rate as a function of the external pumping frequency. The result shown in Figure 5 shows both a broadening and a reduction of the resonant peaks, as one would expect for waves propagation in a more dissipative medium. Our next step was to quantify this enhanced dissipation by fitting the analytical result with $\nu_{\text {eff }}$ as free parameter. The best fit to the numerical results shown in Figure 5 corresponds to

$$
\nu_{e f f} \approx 0.03
$$

which is much larger than the flow viscosity used in our simulations $\nu=\eta=4.10^{-3}$.

\section{Conclusions}

We briefly summarized our current understanding on the problem of coronal heating (see also Aulanier (2011)). Even though there are several theoretical models for coronal heating proposed in the literature, which can be broadly classified as DC or AC, we identified two key assumptions that are common to almost all of these models. Namely, that the ultimate energy source is the kinetic energy of the subphotospheric convection, and the existence of fine scale structures to speed up dissipation.

Within this context, we propose that the development of MHD turbulence in coronal loops efficiently speeds up dissipation of the energy provided by footpoint motions.

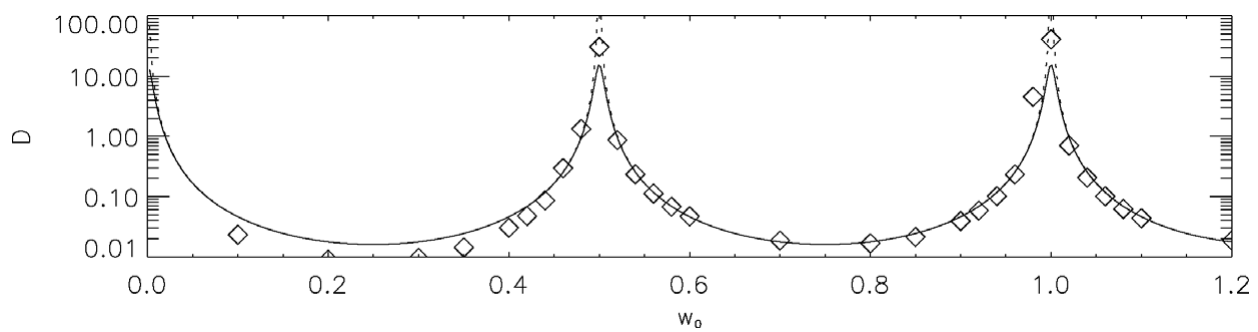

Figure 4. Asymptotic dissipation rate (log-scale) vs. the wave frequency $\omega_{0}$. Diamonds correspond to numerical results, the full trace corresponds to the analytical curve for $\nu=4.10^{-3}$ and the dotted trace corresponds to the ideal limit. 


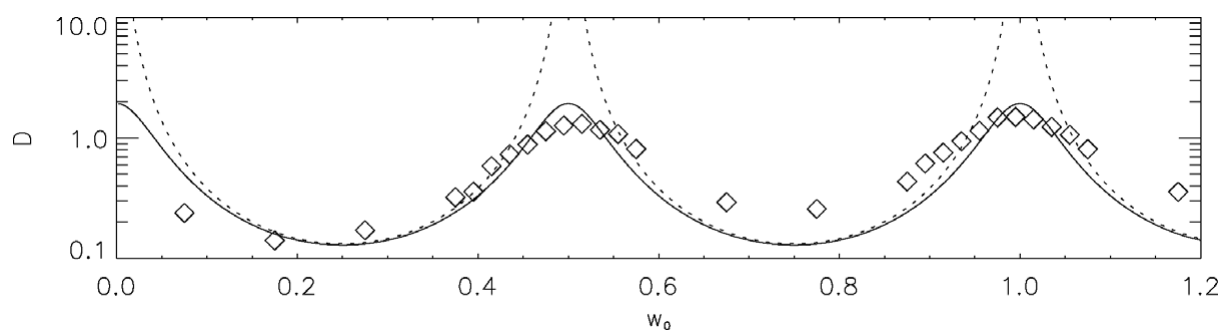

Figure 5. Asymptotic dissipation rate (log-scale) vs. the wave frequency $\omega_{0}$. Diamonds correspond to numerical results with turbulent background, the full trace corresponds to the analytical curve for $\nu_{e f f}=0.03$ and the dotted trace corresponds to the ideal limit.

Turbulent fluctuations of the velocity and magnetic field within the loop, contribute to transfer energy toward small scales due to nonlinear interactions, until highly structured electric currents are formed. Numerical simulations of the RMHD equations show that even slow and stationary footpoint motions develop a turbulent regime after a few photospheric turnover times. Our simulations also show that Alfven waves propagating into coronal loops dissipate much faster in the presence of a turbulent regime.

In summary, the development of fine scales to enhance the dissipation of either waves or DC currents is a natural outcome of turbulence models. Furthermore, the intermittent nature of turbulent dissipation is fully consistent with the highly dynamic behavior displayed in recent observations of coronal loops in solar active regions (see for instance Patsourakos \& Klimchuk (2009)).

\section{References}

Aschwanden, M. J. 2004, in Physics of the Solar Corona. An Introduction, Springer-Verlag, Berlin.

Aulanier, G. 2011, in "Proc. IAU Symp. 273: Physics of the Sun and Star spots", in press.

Demoulin, P., van Driel-Gesztelyi, L., Mandrini, C. H., Klimchuk, J. A., \& Harra, L. 2003, Astrophys. J., 586, 592 .

Dmitruk, P. \& Gómez, D. O. 1997, Astrophys. J., 484, L83.

Dmitruk, P., Gómez, D. O., \& DeLuca, E. 1998, Astrophys. J., 505, 974.

Dmitruk, P. \& Gómez, D. O. 1999, Astrophys. J., 527, L63.

Galsgaard, K. \& Nordlund, A. 1996, J. Geophys. Res., 101, 13445.

Georgoulis, M., Velli, M., \& Einaudi, G. 1998, Astrophys. J., 497, 957.

Gómez, D. O. 1990, Fund. Cosmic Phys., 14, 361.

Gómez, D. O. \& Ferro Fontán, C. 1988, Solar Phys., 11633.

Gómez, D. O. \& Ferro Fontán, C. 1992, Astrophys. J., 394, 662.

Gómez, D. O. \& Dmitruk, P. 2008, in "Proc. IAU Symp. 247: Waves and Oscillations in the Solar Atmosphere", (Eds. R. Erdelyi \& C.A. Mendoza-Briceño), 269.

Gudiksen, B. V. \& Nordlund, A. 2002, Astrophys. J., 572, L113.

Hendrix, D. L. \& van Hoven, G. 1996, Astrophys. J., 467, 887.

Heyvaerts, J. \& Priest, E. R. 1992, Astrophys. J., 390, 297.

Inverarity, G. W. \& Priest, E. R. 1995, Astron. Astrophys, 302, 567.

Longcope, D. W. \& Sudan, R. N. 1994, Astrophys. J., 437, 491.

Mandrini, C. H., Demoulin, P., \& Klimchuk, J. A. 2000, Astrophys. J., 530, 999.

Mikić, Z., Schnack, D. D., \& van Hoven, G. 1989, Astrophys. J., 338, 1148.

Narain, U. \& Ulmschneider, P. 1990, Space Sci. Rev., 54, 377.

Narain, U. \& Ulmschneider, P. 1996, Space Sci. Rev., 75, 453.

Parker, E. N. 1972, Astrophys. J., 174, 499.

Parker, E. N. 1988, Astrophys. J., 330, 474. 
Patsourakos, S. \& Klimchuk, J. A. 2009, Astrophys. J., 696, 760.

Rappazzo, A. F., Velli, M., Einaudi, G., \& Dahlburg, R. B. 2008, Astrophys. J., 677, 1348.

Strauss, H. 1976, Phys. Fluids, 19, 134

van Ballegooijen, A. A. 1986, Astrophys. J., 311, 1001.

Zirker, J. B. 1993, Solar Phys., 148, 43. 